\title{
Optimization Performance Protocol Leach Sensor Network With Multi Sink
}

\author{
Fandi Ali Mustika ${ }^{1}$, Sulistyani Puteri Ramadhani ${ }^{2}$ \\ \{fandi.ali@mercubuana.ac.id ${ }^{1}$, sulistyani@trilogi.ac.id ${ }^{2}$ \} \\ Universitas Mercu Buana, Indonesia ${ }^{1}$, Universitas Trilogi, Indonesia ${ }^{2}$
}

\begin{abstract}
The development of Wireless Sensor Network (WSN) in recent years, researchers are in it. The topics studied by many researchers are energy consumption, coverage and connectivity in WSN. This paper will discuss WSN in the presence of a simple obstacle or resistor. The LEACH protocol algorithm is used and modified in which there is an obstacle or obstacle with multi sink. We propose an algorithm based on line intersections to improve the original LEACH algorithm with multi sink. The simulation results show the modified LEACH algorithm will work better than the original LEACH in the presence of multi-sink based on energy consumption and loss of transmission data. Future research will have arbitrary obstacle modifications.
\end{abstract}

Keywords: protocol LEACH, WSN, multi sink, obstacle.

\section{Introduction}

Wireless Sensor Network (WSN) is a wireless network ad hoc consisting of a number of small devices, known as sensors, and spread over a specific geographic region[1]. Each device has the ability to perform data processing and communicate wirelessly, making it possible to gather information from the surrounding environment and generate and transmit messages to its remote base station. A remote base station, tasked with collecting and analyzing received messages and deciding whether or not an unusual event or a particular phenomenon occurs in the area where the sensor is spread[1].

One of the main advantages of WSN is that WSN does not rely on wiring and constraints budget. In order to retain all the benefits of WSN's wireless operations, each node sensor must be equipped with an inexpensive, ongoing energy source. Therefore, each node sensor uses a small battery as its power source. While activities such as recharging batteries or replacing batteries in remote and far-reaching areas, where the nodes sensor are located, are not easy to do.

Jeong explains, one of the main problems in power management is how to schedule the sensors to obtain longer network life time but still able to meet the high quality of service requested[2]. Energy efficient design to extend WSN's life without sacrificing system reliability is an important challenge for the design of a wider wireless sensor network.

One of the advantages of WSN is its ability to work in an environment where it is difficult for humans to enter it due to risk, inefficient or sometimes impossible. Therefore, the sensor is expected to replace the monitoring through humans, which is randomly distributed in the area of observation. This random spread can be done by throwing it from the helicopter, which in the field later, these sensors can work together ad ICCSET 2018, October 25-26, Kudus, Indonesia

Copyright (C) 2018 EAI

DOI 10.4108/eai.24-10-2018.2280585 
hoc. Under these conditions, things that often happen are, the possibility of damaged sensors and sensors that have a short life time (due to low energy levels). Therefore, designing and operating this huge network requires scalable management and architectural strategies. Because of the limited energy levels and batteries of these nodes can't be recharged, efficient design of algorithms in energy use is critical to extending the viability of these sensors[1].

A widely used strategy is to combine nodes into a cluster. Each cluster has leader, called cluster head $(\mathrm{CH})$. One of the cluster algorithms used in WSN is LEACH. LEACH in WSN is a protocol that governs the concept of clustering for the nodes used. The presence of attenuation of the obstacle will affect the range of communication between sensor nodes due to the Occurrence of power lost during transmission[3].

Spread of nodes sensor on WSN is still an interesting topic for researchers. Good spread of sensors should consider good coverage and connectivity areas. Coverage requires that each sensory field location be monitored by at least one sensor. Connectivity requires that the network is not fragmented in the communication node. The provision that needs to be considered is that coverage is influenced by the sensitivity level of the sensor, while connectivity is influenced by the communication of the sensor node[4].

In addition to limited energy, there are other characteristics of WSN which make them different from other wireless networks. First, due to large number of sensor nodes in WSN, scalability related issues arise in it. Second, due to small size of sensor nodes, WSN have small bandwidth, limited computing and communicating capabilities. Finally, sensor nodes broadcast their data, so there is high probability of data redundancy. These characteristics propose many research challenges to study of sensor networks in each layer[5].

Obstacles such as walls, buildings, house blocks or unexpected obstacles often lie within the senses region. The obstacle significantly affects the coverage and connectivity of the sensor node which means it will also affect the shape of the solution of the sensor deployment. The presence of attenuation of the obstacle will affect the range of communication between sensor nodes due to the occurrence of power lost during transmission.

The virtue of this research is the design of topology or sensor network infrastructure to be used in consuming energy efficient in its use in every wireless sensor in real time.

\section{Related Work}

Before discussing the high-level classification of energy conservation proposals, it is worth presenting the network and node-level architecture we will refer to. From a sensor network standpoint, we mainly consider the model depicted in figure 1 , which is the most widely adopted model in the literature. On the other side, figure 2 shows the architecture of a typical wireless sensor node, as usually assumed in the literature. . 


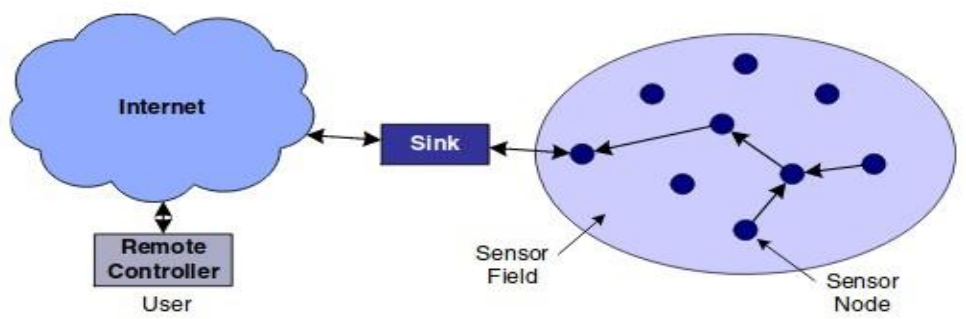

Fig. 1. Sensor Network Architecture.

However, as the latter components are optional and only occasionally used, we will not take them into account in the following discussion. Obviously, the power breakdown heavily depends on the specific node. In[6] it is shown that the power characteristics of a Mote-class node are completely different from those of a start gate node. However, the following remarks generally hold[6]

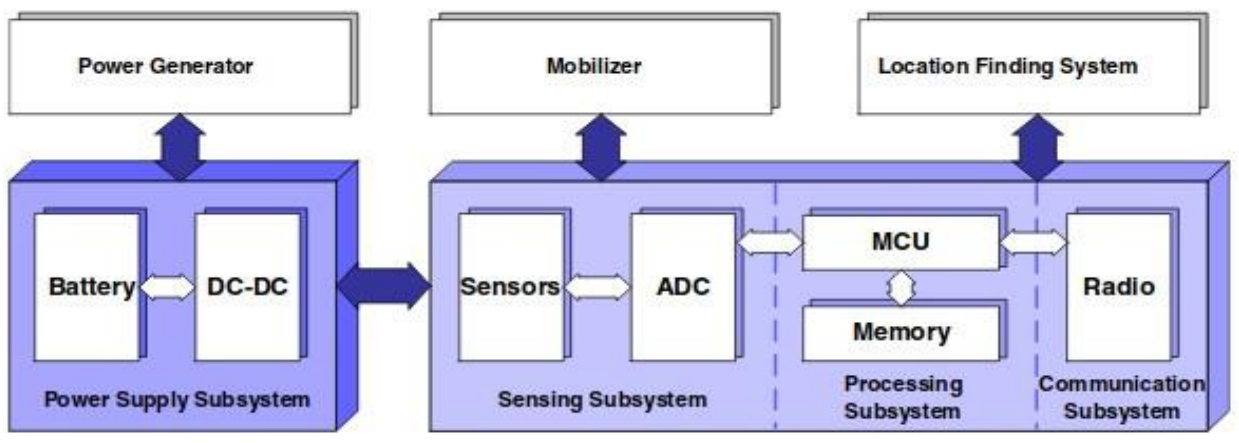

Fig. 2. Architecture of a typical Wireless Sensor Network.

The communication subsystem has an energy consumption much higher than the computation subsystem. It has been shown that transmitting one bit may consume as much as executing a few thousands instructions. Therefore, communication should be traded for computation.

The radio energy consumption is of the same order of magnitude in the reception, transmission, and idle states, while the power consumption drops of at least one order of magnitude in the sleep state. Therefore, the radio should be put to sleep (or turned off) whenever possible.

Depending on the specific application, the sensing subsystem might be another significant source of energy consumption, so its power consumption has to be reduced as well.

We have provided discussions and recommendations concerning energy consumption and communication protocol for this monitoring sensor networks. 


\section{Research Method}

In carrying out a study, a method is required which is the stages and activities that are part of the research roadmap. Researchers in conducting their research will use the model and simulation methods that can be seen in Figure 3.

The use of model and simulation method is well known in the world of engineering techniques. This method helps reduce costs and improve system quality.

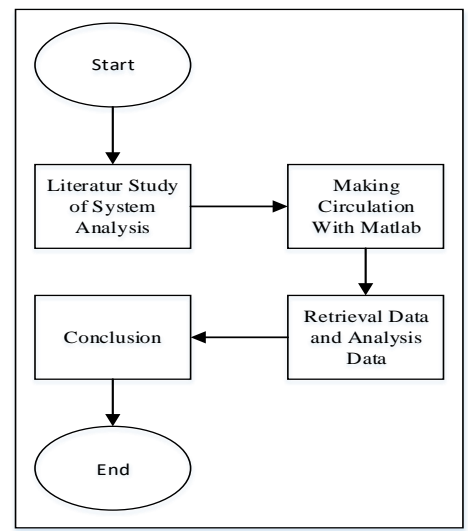

Fig. 3. Research Method

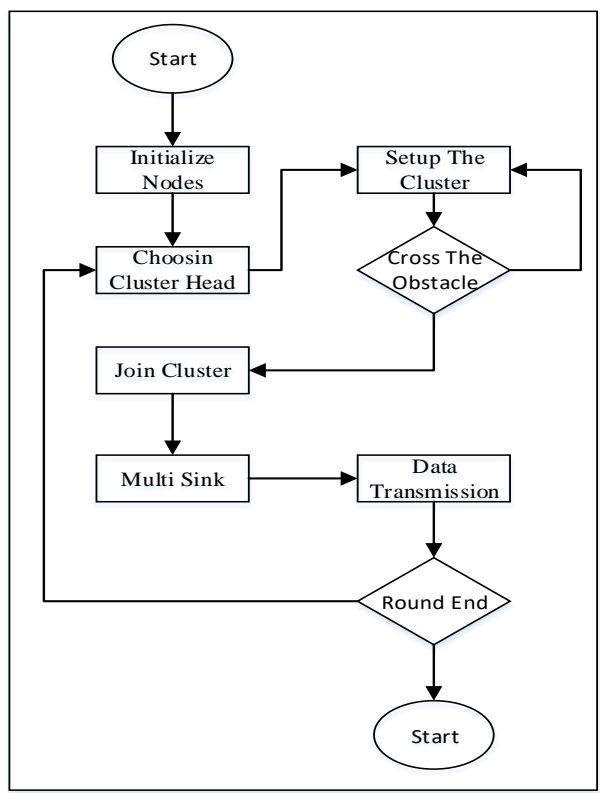

Fig. 4. Flowchart Modification of LEACH 
Modification of the LEACH algorithm proposed in this study focuses on checking obstacle and multi sink. This algorithm runs with the node when the node will be associated with Cluster Head $(\mathrm{CH})$.

\subsection{Model Making}

The model is defined as a representation or abstraction of an actual object or situation[8]. Models can be classified into iconic, analogic and symbolic models. The iconic model is to present a system or object into an object model whose foresees that system. An analog model is a model capable of presenting the nature of a system to be simpler. The symbolic model or mathematical model is an abstract representation of a system.

In this study, modeling will produce a model system that is a representation of the real state but modeled into a manageable format with the computer.

\subsection{Simulation Design}

Design is the next step. At this stage, the research is continued by utilizing the model system that has been generated from the previous stage, is modeling.

Simulation is an imitation of the operating process of a real condition or system over time. Simulation are used to describe and analyze the behavior of a system, ask what if ("what if") question about real systems, and assist in the design of real systems. Simulation refers to a broad set of methods and application of behavioral imaging of the real system. The simulation model is an appropriate alternative in describing a complex system, especially when a analytical mathematical model is difficult to perform.

\subsection{Performance Indicators Analysis}

A two approaches can be used to measure the performance of a networks, is by performing real test bed or by simulation modeling. The advantage of doing a real test bed is to uncover problems that cannot be detected by simulation. But the test bed cannot be used for large scale. With the simulation, learning about the behavior of network systems becomes easier, because it uses the simplification of various parameters. Furthermore, for networks of larger size can be made possible by simulation.

An obstacle, in this study is rectangular, and only becomes obstacle in the created scenario. In fact, the position of the obstacle is fixed and unchanged. We describe an obstacle with a rectangular line and line-shaped signal transmission as well as illustrated in Figure 5.

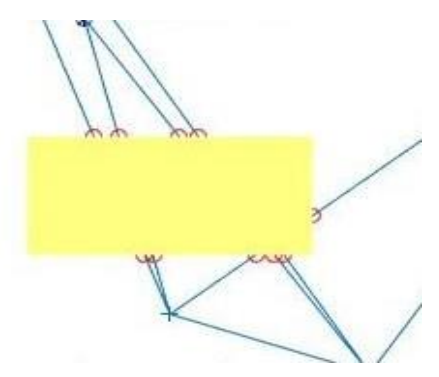

Fig. 5. Obstacle and Transmission Line. 
For the first node $a\left(x_{1}, y_{1}\right)$ and the second node $b\left(x_{2}, y_{2}\right)$, if $a$ transmission goes to $b$, then the signal line will pass obstacle $o\left(\left(x_{3}, y_{3}\right),\left(x_{4}, y_{4}\right)\right)$. To get the junction we use the equation of two lines.

$$
\left(\mathrm{x}-\mathrm{x}_{1}\right)\left(\mathrm{y}_{2}-\mathrm{y}_{1}\right)=\left(\mathrm{y}-\mathrm{y}_{1}\right)\left(\mathrm{x}_{2}-\mathrm{x}_{1}\right)
$$

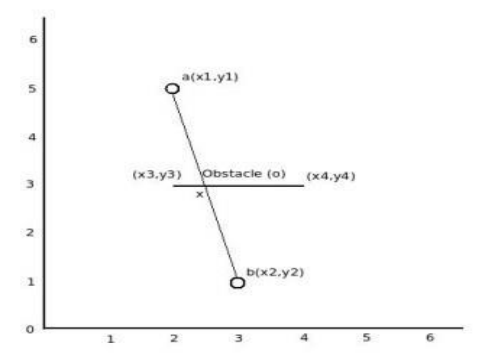

Fig. 6. Obstacle Model between Two Lines.

\section{Simulation and Result}

\subsection{Simulation}

Parameters the simulation parameters for this study are described in Table 1.

\subsection{Simulation Case}

In this section, we will provide three cases that will be implemented in the simulation. The first case, obstacle a simple is placed in a simulation with the original LEACH algorithm. The second case, the obstacle is placed between several nodes that surround it. The third case, the obstacle is placed between nodes that have been deployed with multiple sinks.

In the first case, obstacle we do not create and give four nodes. Figure 7, we will show the first case simulation. In the second case, the obstacle is rectangular and its size is $12 \times 2 \mathrm{~m}$. There is some space for the nodes to interact to the BS (Base Station). Figure 8, will show the second case simulation. In the third case, the length of the obstacle is lengthened to $28 \mathrm{x} 1 \mathrm{~m}$. Among the nodes that will interact to some BS to be placed. The third simulation case this will be illustrated in figure 9 . 


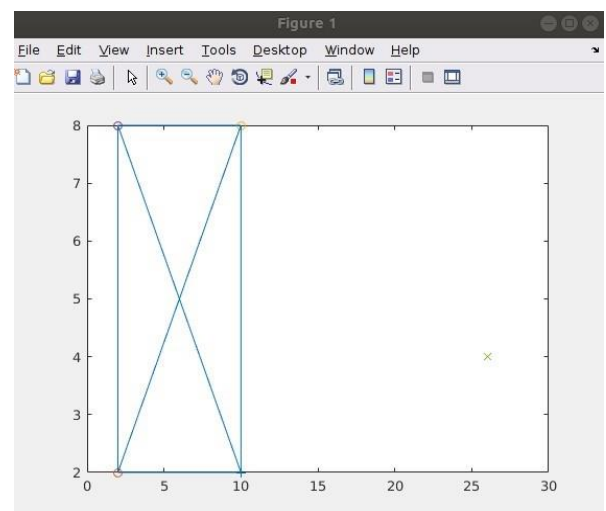

Fig. 7. First Case Simulation.

Table 1. Parameters Simulation

\begin{tabular}{lc}
\hline \multicolumn{1}{c}{ Parameter } & Value \\
\hline Simulation Area of Nodes & $20 \times 20 \mathrm{~m}$ \\
Number of Nodes & $4-6$ \\
Initial Energy & $0.5 \mathrm{~J}$ \\
Energy Aggregation Data & $5 \mathrm{~nJ} / \mathrm{bit}$ \\
EElec & $50 \mathrm{~nJ} / \mathrm{bit}$ \\
EFS & $10 \mathrm{pJ} / \mathrm{bit} / \mathrm{m}^{2}$ \\
Emp & $0.0012 \mathrm{pJ} / \mathrm{bit}^{2} / \mathrm{m}^{2}$ \\
Cluster Head Prob. & 0.1 \\
Heterogeneity & 0.4 \\
Routing Algorithm & $\mathrm{LEACH}$ \\
Simulation Round Time & 100 \\
Data Transmission & $1 \mathrm{bps}$ \\
\hline
\end{tabular}

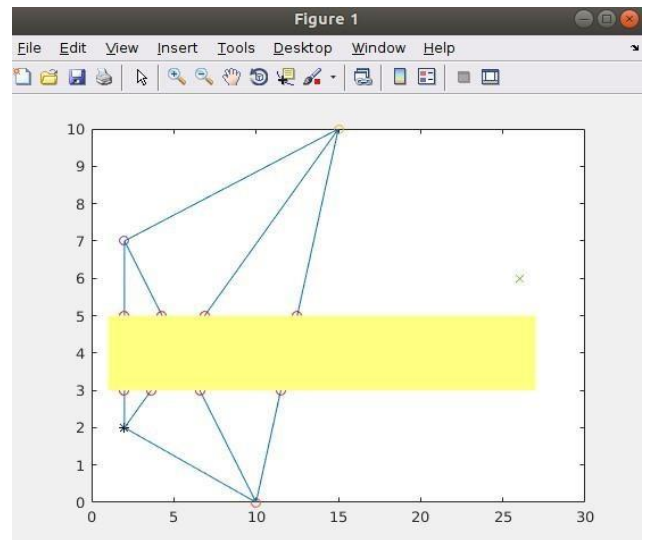

Fig. 8. Second Case Simulation. 


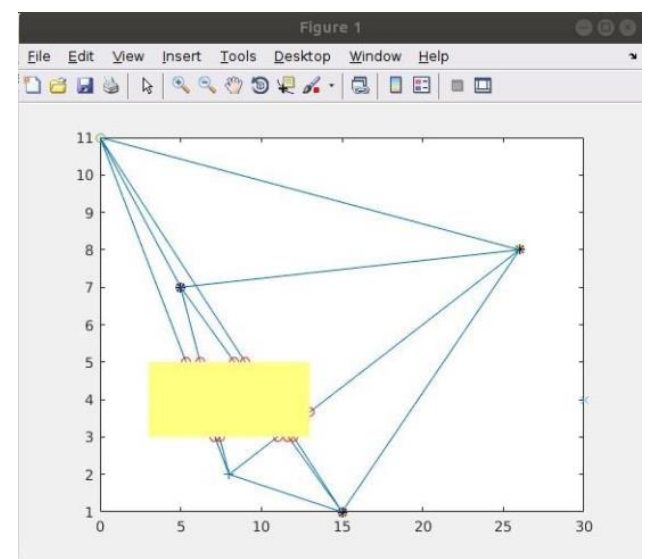

Fig. 9. Third Case Simulation.

\subsection{Simulation Result}

We will show the results of a combined simulation of the three case in one result where to show the energy efficiency of the three case made. Figure 10 show the average remaining energy for all nodes.

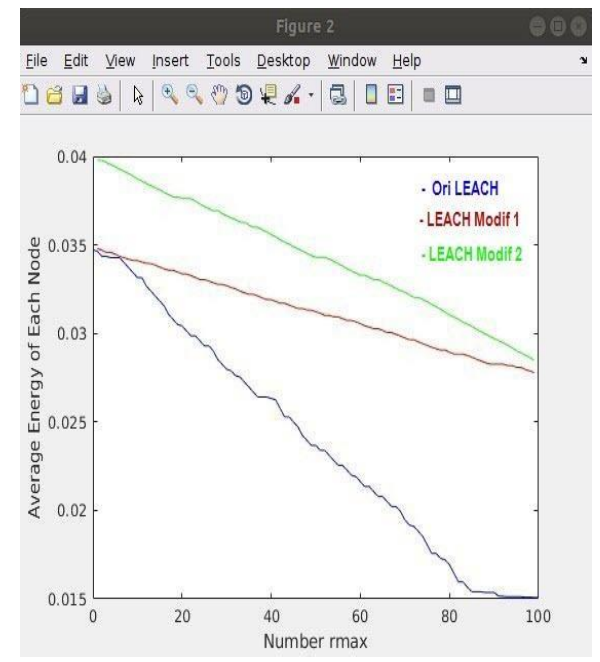

Fig. 10. The Average Residual Energy of All Nodes.

From the results of figure 8 , there are several lines to be explained that the blue line is the result of the remaining energy from the first case of the original LEACH. For the red line represents the line from the second case energy yield of the LEACH modification which results in the average remaining energy and the green color is the average residual energy yield in the third case will be shown in figure 8. 
The original LEACH algorithm consumes more energy because a collection of $\mathrm{CH}$ nodes always tries to communicate to all the nodes inside $\mathrm{CH}$. We can see the remaining percentage of energy for original LEACH in the first case of $99.23 \%$, in the second case the remaining energy is $47.33 \%$, and in the third case the remaining energy is $53.45 \%$, listed in Table 2 .

Table 2. Average Energy.

\begin{tabular}{lll}
\hline \multicolumn{1}{c}{ Case } & Result Average & Energy Percentage of Remaining Energy \\
\hline First & 0.01523 & $99.23 \%$ \\
Second & 0.28343 & $47.33 \%$ \\
Third & 0.32222 & $53.45 \%$ \\
\hline
\end{tabular}

Energy consumption in second and third case is slightly higher than in the first case. This happens because, there are some nodes that cannot communicate with the BS. To prove the case, the matrix is likely to be show in the table 3 .

Base on table 3, the value of the matrix in BS, the column for node 1 and 2 (with an asterisk) is 1 . A value of 1 indicated the existence of obstacle between BS and $\mathrm{CH}$. And a value of 0 indicated there is no obstacle between $\mathrm{BS}$ and $\mathrm{CH}$.

Table 3. Matrix Possibility of Second and Third Case.

\begin{tabular}{ccccc}
\hline Node & 1 & 2 & 3 & 4 \\
\hline 1 & & 1 & 1 & 0 \\
2 & 1 & & 1 & 0 \\
3 & 1 & 0 & & 0 \\
4 & 1 & 1 & 0 & \\
BS & $* 1$ & $* 1$ & 0 & 0 \\
\hline
\end{tabular}

The second parameter of the second and third cases is the loss of transmission data. The average transmission loss data will be show in the table 4 . The average transmission loss data for the second and third cases is greater than the first case. Because there are some nodes that cannot communicate to the BS because it is separate from obstacle.

Table 4. Average Data Output Missing.

\begin{tabular}{cc}
\hline Original LEACH & Value \\
\hline Average Loss of Transmission Data & $190 \mathrm{bps}$ \\
\hline
\end{tabular}

\section{Conclusion}

Wireless sensor network with multi sink has some drawbacks. One example is to consume energy faster than other nodes. Traffic patterns in wireless sensor networks are usually manyto-one models. Learning from the concept, the virtual hybrid potential of the wireless sensor network has been built based on hops and the remaining energy from each node. Therefore, by consuming faster energies, multi sink are created to minimize the excessive use of energy in terms of reach and connectivity. 


\section{References}

[1] G. Xu, W. Shen, and X. Wang, "Applications of wireless sensor networks in marine environment monitoring: A survey," Sensors (Switzerland), vol. 14, no. 9, pp. 16932-16954, 2014.

[2] C. Y. Chang, K. P. Shih, S. C. Lee, and S. W. Chang, "RGP: Active route guiding protocol for wireless sensor networks with obstacles," 2006 IEEE Int. Conf. Mob. Ad Hoc Sens. Syst. MASS, vol. 1, pp. 367-376, 2006.

[3] A. Wahab, F. A. Mustika, R. B. Bahaweres, D. Setiawan, and M. Alaydrus, "Energy efficiency and loss of transmission data on Wireless Sensor Network with obstacle," in Proceeding of 2016 10th International Conference on Telecommunication Systems Services and Applications, TSSA 2016: Special Issue in Radar Technology, 2017.

[4] S. Meguerdichian, F. Koushanfar, G. Qu, and M. Potkonjak, "Exposure in wireless Ad-Hoc sensor networks," Proc. 7th Annu. Int. Conf. Mob. Comput. Netw. - MobiCom '01, pp. 139-150, 2001.

[5] A. Syarif, A. Abouaissa, L. Idoumghar, R. F. Sari, and P. Lorenz, "Performance analysis of evolutionary multi-objective based approach for deployment of wireless sensor network with the presence of fixed obstacles," 2014 IEEE Glob. Commun. Conf. GLOBECOM 2014, pp. 1-6, 2014.

[6] V. Raghunathan, C. Schurgers, S. Park, and M. B. Srivastava, "Energy-aware wireless microsensor networks," IEEE Signal Process. Mag., vol. 19, no. 2, pp. 40-50, 2002.

[7] R. J. Mohsin, J. Woods, and M. Q. Shawkat, "(AMDC) Algorithm for wireless sensor networks in the marine environment,” IJACSA) Int. J. Adv. Comput. Sci. Appl., vol. 6, no. 6, 2015.

[8] A. Syarif, I. Benyahia, A. Abouaissa, L. Idoumghar, R. F. Sari, and P. Lorenz, "Evolutionary Multi-Objective Based Approach for Wireless Sensor Network Deployment."

[9] Albaladejo C, Soto F, Torres R, Sánchez P and López J A 2012 A low-cost sensor buoy system for monitoring shallow marine environments Sensors (Switzerland) 12 9613-34

[10] F.J. Velez, A. Nadziejko, A.L. Christensen, S. Oliviera, "Wireless Sensor and Networking Technologies for Swarms of Aquatic Surface Drones", IEEE 82nd Vehicular Technology Conference (VTC Fall), 6-9 September 2015, Boston, USA, DOI: 10.1109/VTCFall.2015.7391193

[11] B. Manzoor, N.Javaid, O. Rehman, M. Akbar, Q. Nadeem, A. Iqbal, M. Ishfaq, "Q-LEACH: A New Routing Protocol for WSNs", Procedia Computer Science, Vol. 19, pp. 926-931, 2013. 